\title{
Peroral esophageal segmentectomy and anastomosis with single transthoracic trocar: a step forward in thoracic NOTES
}

Authors

Institutions
C. Rolanda ${ }^{1,2}$, D. Silva ${ }^{1,3}$, C. Branco ${ }^{1}$, I. Moreira ${ }^{4}$, G. Macedo ${ }^{5}$, J. Correia-Pinto ${ }^{1,6}$

Institutions are listed at the end of article. submitted 2 January 2010 accepted after revision 12 August 2010

\section{Bibliography}

Dol http://dx.doi.org/ 10.1055/s-0030-1256012 Endoscopy 2011; 43: 14-20 @ Georg Thieme Verlag KG Stuttgart · New York ISSN 0013-726X

\section{Corresponding author} J. Correia-Pinto, MD PhD Instituto de Ciências da Vida e Saúde

Escola de Ciências da Saúde Universidade do Minho, Campus de Gualtar 4709-057 Braga

Portugal

Fax: +351-253-604831

jcp@ecsaude.uminho.pt
Background and study aims: A transesophageal natural orifice transluminal endoscopic surgery (NOTES) approach has been proposed for thoracic and mediastinal access. Similarly to transgastric surgery, serious limitations remain related to creating an esophagotomy and its safe closure. A hybrid approach in thoracic NOTES could work as an intermediate step before pure transesophageal NOTES. We assessed the benefit of hybrid thoracic NOTES for peroral segmental esophagectomy and subsequent complete esophageal anastomosis with a single transthoracic port.

Methods: Two protocols were used to attempt esophago-esophageal anastomosis: ex vivo using a phantom model $(n=5)$, and in vivo after esophageal mobilization, and segmental esophagectomy achieved using either a gastroscope (flexible) $(n=5)$ or thoracoscope (rigid) instruments $(n=5)$. A forward-viewing double-channel endoscope

\section{Introduction}

\section{$\nabla$}

A minimally invasive esophageal approach is being used more frequently for both benign and malignant esophageal disease [1]. Since the first thoracoscopic mobilization of the esophagus described by Cuschieri in 1992, different surgical techniques have been introduced [2,3]. However, it should be emphasized that thoracic incisions even if small are painful [4].

With natural orifice transluminal endoscopic surgery (NOTES) [5-8], a new approach to the thorax and mediastinum has been proposed: transesophageal access [9]. Although there are descriptions of transvesical transdiaphragmatic thoracoscopy [10] and transgastric transdiaphragmatic pericardial fenestration, the most reasonable reported thoracic access is the transesophageal. If the esophagus was used as an entry site into the chest, a direct access to the thorax and posterior mediastinum could reasonably be established [11]. In fact, the technical feasibility of a transeso- and a transthoracic operative thoracoscope with a working channel were coordinated in order to create a complete single-layer, end-to-end esophageal anastomosis ex vivo as well as in vivo. Feasibility and anastomosis quality were evaluated by inside and outside assessment of: patency, the incorporation of mucosa in all stitches, and a leak test.

Results: Anastomosis was achieved in all ex vivo experiments and thoracoscopically-led in vivo procedures. All anastomoses were patent, allowing distal passage of the endoscope, with mucosa incorporation. In in vivo experiments, a leak was detected in three animals and corrected with additional stitching.

Conclusions: Peroral esophageal anastomosis with a single transthoracic trocar is feasible, which may represent a step forward in thoracic NOTES.

phageal approach has been described in porcine models for several simple thoracic procedures [11-16]. Even if the general concept is appealing, the transesophageal approach seems the one that most stretches the limits, even in the NOTES world. Access by the transesophageal approach may be highly risky, and mechanical abrasion and trauma of surrounding structures is higher when compared with the other visceral access routes. Moreover, the potential hemodynamic compromise due to an uncontrolled pneumomediastinum, and the consequences of an ineffective esophagotomy closure can be devastating for the patient, because of the morbid consequences of mediastinitis. In this sequence, the well-known problems of NOTES [17], such as safe enterotomy creation, infection prevention, tissue manipulation, and, primarily, suturing and the establishment of anastomosis, seem to be even more limiting in the transesophageal approach.

Mirroring what is being done in abdominal surgery, where the hybrid approach became a transi- 
tional step for humans in reducing the number of transabdominal ports and enabling a natural orifice approach without losing safety $[18,19]$, we hypothesized that the use of a peroral approach in a hybrid mode can significantly reduce the number of transthoracic ports.

Aiming to eliminate some of the current limitations of transesophageal NOTES, we designed this study to carry out peroral esophageal mobilization, segmental esophagectomy and esophago-esophageal anastomosis with a single trocar, to assess the reliability of this strategy for opening, resection and suturing of the esophagus.

This project was funded by the following grants: Bolsa de Investigação da Sociedade Portuguesa de Endoscopia Digestiva 2007 and the FCT project - PTDC/SAU-OSM/105578/2008.

\section{Materials and methods}

$\nabla$

\section{Study design}

This study was approved by ethical review boards of Minho University (Braga, Portugal).

The study was divided in two main branches: ex vivo studies in which esophago-esophageal anastomoses were carried out in a phantom model (five porcine esophaguses), and in vivo studies in anesthetized pigs. The in vivo studies involved esophageal mobilization and segmental esophagectomy using either a flexible gastroscope (five animals) or rigid instruments introduced through the working channel of an operative thoracoscope (five animals). Esophago-esophageal anastomoses were then carried out in the subset group in which esophagectomy had been carried out using rigid instruments introduced through the working channel of an operative thoracoscope. In all conditions, we used a forward-viewing double-channel gastroscope (G28/34; Karl Storz, Tuttlingen, Germany) and a transthoracic 10 -mm operative thoracoscope with a 5-mm working channel (26036AA; Karl Storz).

\section{Ex vivo studies}

Phantom model A three-dimensional structure consisting of synthetic polyurethane foam with an attached PVC container was mounted to simulate the thoracic cavity, and a tube $20 \mathrm{~cm}$ long was used to reproduce the oropharynx. Esophaguses harvested from adult pigs were positioned, fixed and sectioned in the middle segment, creating a gap $4 \mathrm{~cm}$ long between the ends ( $\bullet$ Fig. 1 a). The model had a black, nontransparent removable cover with an opening on the right side to position the trocar (॰ Fig. 1 b).

Surgical technique The operative thoracoscope was inserted into the phantom model through a 12-mm trocar (Excel port; Ethicon Endo-Surgery, Cincinnati, USA), whereas the gastroscope was introduced through the proximal esophagus; both instruments were coordinated to carry out an esophago-esophageal anastomosis ( Fig. 2).

First, a suturing needle with a 3-0 absorbable PDS stitch (Polydioxanone; Ethicon Endo-Surgery) was mounted on a needleholder (26178KPL; Karl Storz), previously passed through the operative thoracoscope working channel and introduced into the phantom model. Then the gastroscope was moved towards the free lower extremity of the esophagus to grasp it on its posterior wall, including always muscle and mucosa, making proximal traction for the first stitch passage (inside to outside). The needle was then repositioned by the needle-holder for approaching the
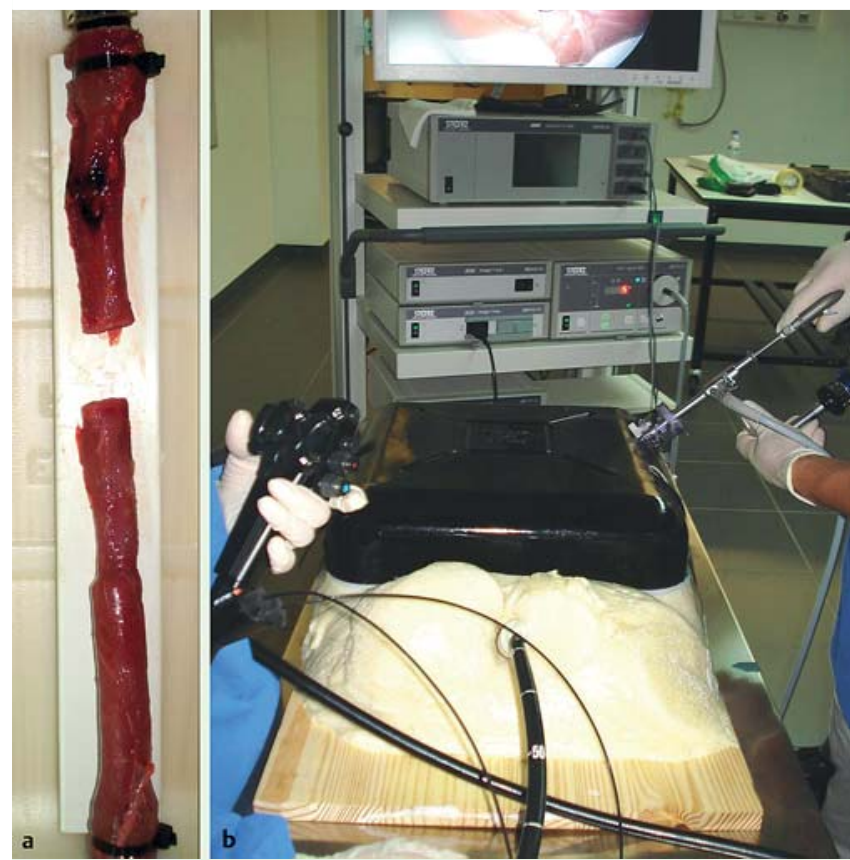

Fig. 1 Phantom model built to simulate the thoracic cavity: a porcine esophagus sectioned in its middle segment and positioned inside the phantom model; b phantom model with its cover.

esophageal upper extremity, and the gastroscope was used to promote esophageal stump alignment and esophageal wall traction for outside-to-in proximal puncture. The knot-tying was achieved by extracorporeal knot techniques using a knot-pusher (26596D; Karl Storz) under gastroscopic vision. Then scissors (34410MW; Karl Storz) were introduced through the operative thoracoscope working channel and the excess suture lengths were cut. This procedure was repeated until 10 single-layer interrupted sutures had been completed, the posterior stitches with internal knotting and the later ones with external knotting. The duration of the procedure was recorded.

When the sutures were complete, the gastroscope was used to verify the immediate endoluminal reliability of the anastomosis, confirming that mucosa from both tips were touching each other, and identifying possible sites for supplementary stitches.

\section{In vivo studies}

Pig preparation Male $40-50 \mathrm{~kg}$ pigs (Sus scrofus domesticus) were fed liquids for 1 day and not allowed food and water for 8 hours before the surgery. All procedures were performed under general anesthesia with endotracheal intubation and mechanical ventilation, according to previous descriptions [20,21]. Before the procedure, $1 \mathrm{mg}$ of intravenous atropine was administered.

Surgical technique The pig was placed in the prone position ( Fig. 3). A 12-mm trocar was positioned around the eighth intercostal space in the right posterior axillary line, through which the operative thoracoscope was introduced; $\mathrm{CO}_{2}$ insufflation was maintained with a pressure up to $6 \mathrm{mmHg}$. The gastroscope was advanced through an oropharyngeal overtube (US Endoscopy, Mentor, Ohio, USA) into the esophagus. Esophageal mobilization and segmental esophagectomy were carried out in the proximal third using either rigid instruments through an operative thoracoscope (five animals) or flexible instruments through a gastroscope (five animals), as detailed schematically in $\bullet$ Fig. 4. 


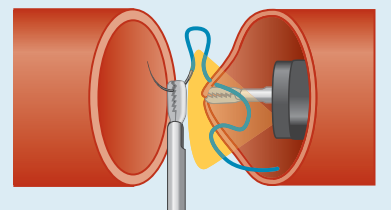

a
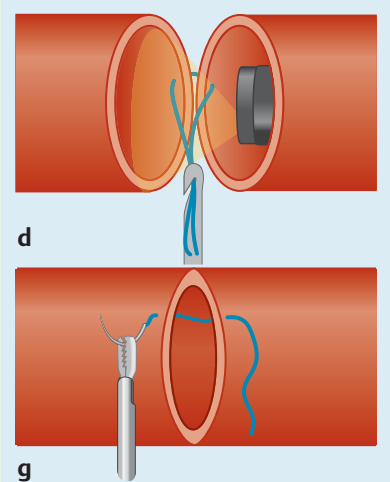

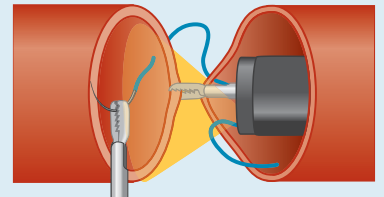

b
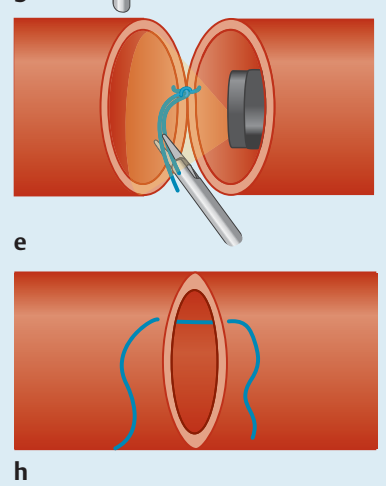

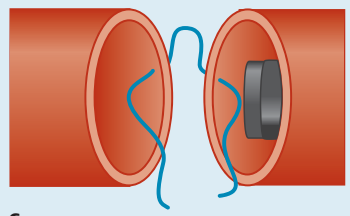

C

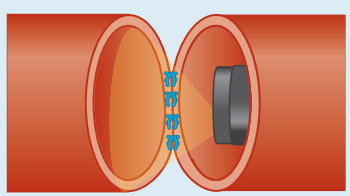

f

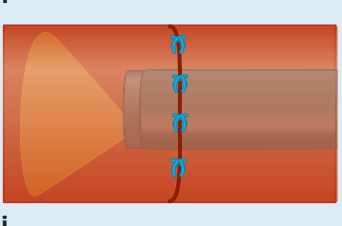

Fig. 2 Steps in esophageal anastomosis: a first stitch with the gastroscope grasper pulling the esophageal wall distally; $\mathbf{b}$ first stitch with the gastroscope grasper pushing the esophageal wall proximally; c first stitch crossed; d knot-pusher closing the first stitch under gastroscope image control; e operative thoracoscope scissors cutting the suture; $\mathbf{f}$ posterior esophageal wall anastomosis complete with inside knotting; $\mathbf{g}$ - $\mathbf{h}$ stitching the anterior wall; i anterior esophageal wall anastomosis complete with outside knotting.

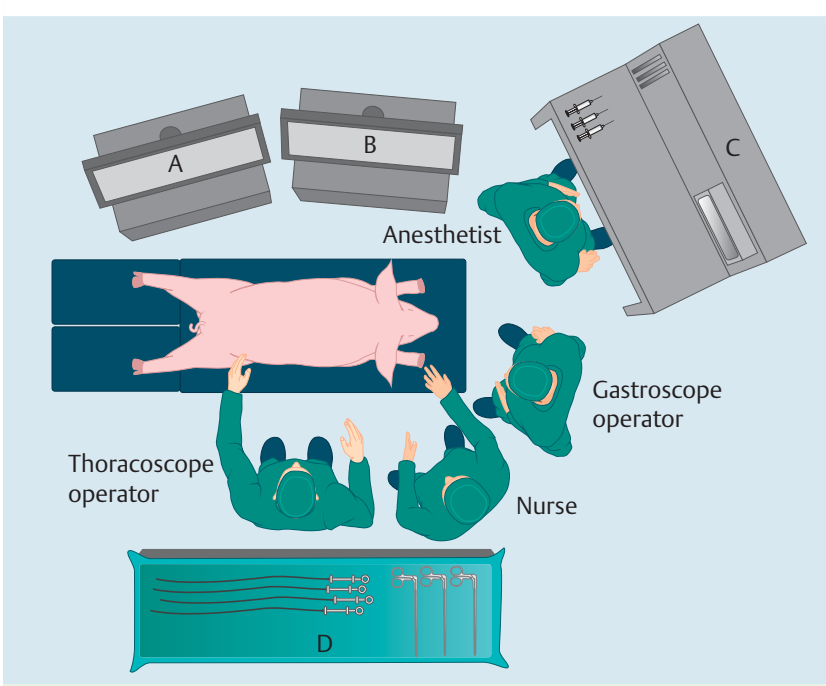

Fig. 3 Layout of the room: A, thoracoscope monitor; B, gastroscope monitor; $C$, ventilator; $D$, back table for equipment.

In those animals where esophagectomy was performed using flexible instruments, the proximal section was carried out with an endoscopic submucosal dissection (ESD) knife. This procedure was enhanced by clamping the esophageal lumen with an external grasper introduced through the working channel of the operative thoracoscope, which helped also in mobilizing the esophagus and providing traction while the gastroscope knife sectioned the esophagus circumferentially with cautery. Subsequently, the gastroscope was introduced into the mediastinum, and the esophagus was released from its vessels and attachments using a flexible hemostatic grasper. Finally, the esophagectomy was completed using an endoscopic snare.

In those animals where esophagectomy was performed with transthoracic instruments, scissors were used through the operative thoracoscope working channel. Under endoluminal traction, the proximal esophagus was dissected, mobilized, freed from its attachments and sectioned.

In both approaches, approximately $4 \mathrm{~cm}$ of esophagus was sectioned and removed perorally.

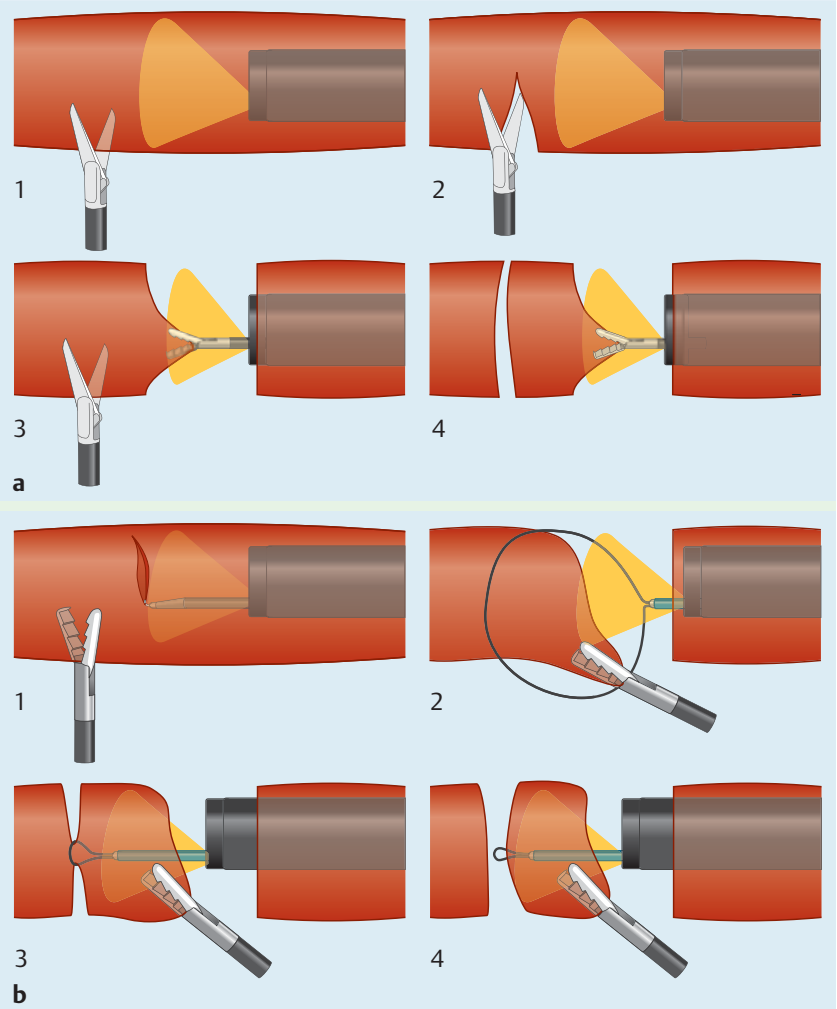

Fig. 4 a Peroral esophagectomy led by the operative thoracoscope. 1 and 2: gastroscope transillumination, thoracoscope positioning and transverse section with rigid scissors. 3 and 4: gastroscopic traction of the distal stump and again transverse section and esophageal segment release by the thoracoscope. $\mathbf{b}$ Peroral esophagectomy led by the gastroscope. 1: thoracoscope fixing, and use of the flexible endoscopic submucosal dissection (ESD) knife to cut the esophagus. 2-4: thoracoscopic traction of the distal stump through a flexible snare; snare section with cautery and esophageal segment release.

In those animals where the esophagectomy was accomplished with the rigid instruments, the gastroscope was reintroduced into the thoracic cavity providing traction and alignment of both esophageal stumps for suturing. The creation of the anastomosis followed the same steps as described in the ex vivo studies ( $\bullet$ Fig. 2). At the end of the procedure, the gastroscope con- 

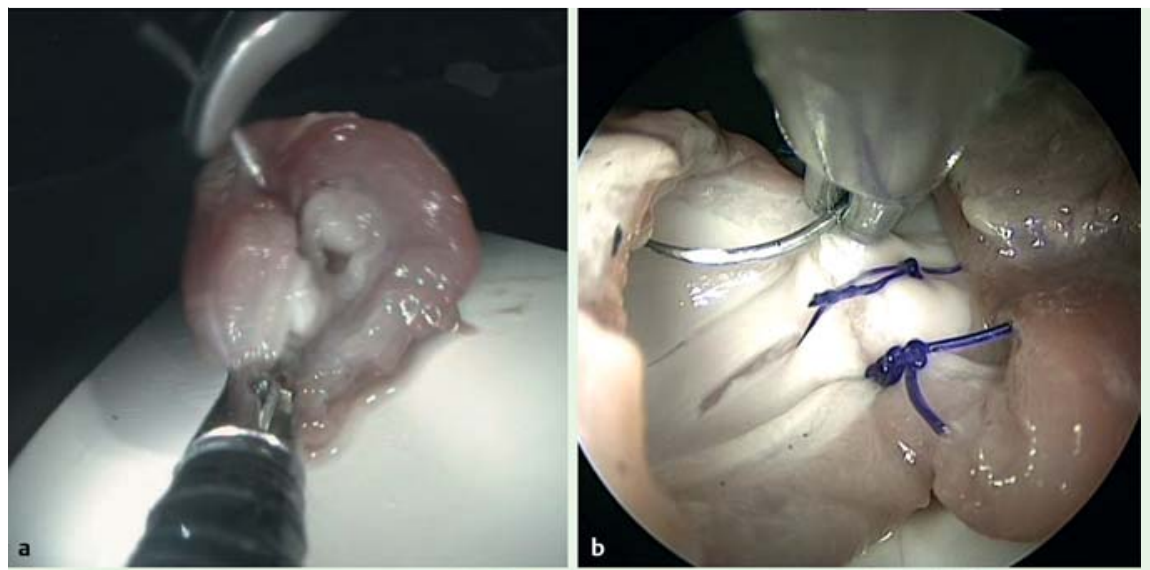

Fig. 5 Ex vivo steps of a complete single-layer, end-to-end esophago-esophageal anastomosis: a grasping, positioning and traction of the distal esophageal extremity provided by the gastroscope for the first stitch passage (gastroscope view); b sequential sutures and needle position for proximal extremity puncture (thoracoscope view); c knot-tying with the knot-pusher entrance monitored by the gastroscope image (gastroscope view); d cutting of excess suture material by the telescopic scissors (thoracoscope view).
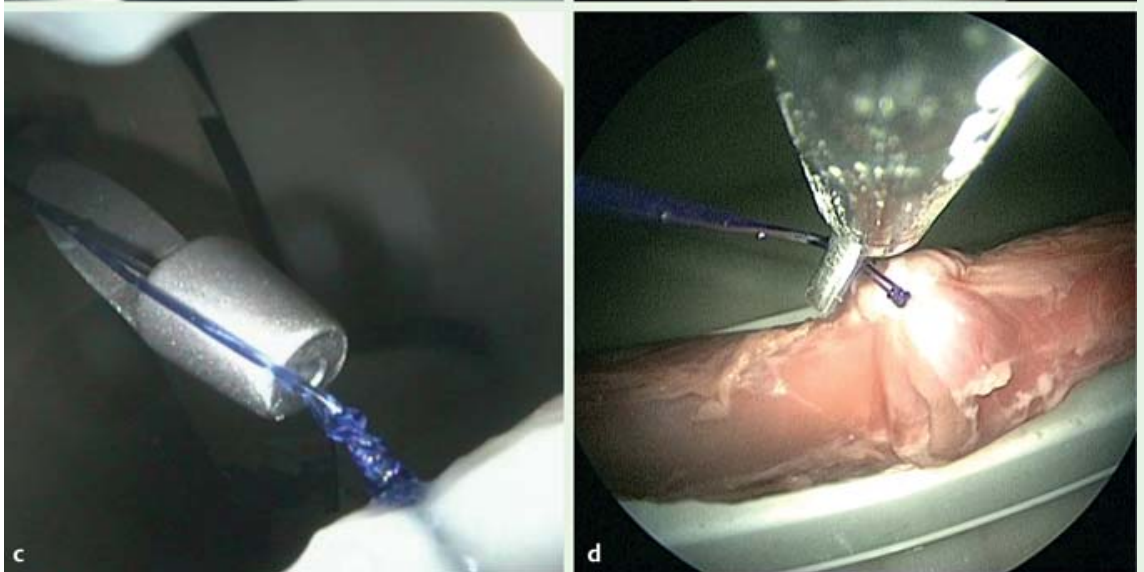

firmed the patency of the anastomosis and the incorporation of mucosa in all stitches; the thoracoscopic view was used to check the reliability of the anastomosis by looking for bubbles released under saline when the gastroscope was insufflating air inside the esophagus (air leak test).

\section{Results}

\section{$\nabla$}

\section{Ex vivo studies}

The phantom model was easy to operate and simulated reasonably well the steps of the the in vivo anastomosis, although the lung, other thoracic structures, and the cardio-respiratory movements were not present.

After some initial difficulties, we verified progressive coordination, making possible the construction of a complete single-layer end-to-end esophageal anastomosis in all experiments, with at least 10 interrupted sutures having five knots each. The crucial step was always the first stitch. Gastroscopic grasping of the muscular and mucosal layers of the distal stump was easily achieved and useful as it provided positioning and traction for the needle passage ( $\bullet$ Fig. 5 a).

Similarly, the gastroscope was useful in exposing the proximal extremity and for countertraction. Once the needle had crossed both extremities, the gastroscope view was also effective in monitoring the knotting. The subsequent stitches were progressively easier to apply ( Fig. $\mathbf{5 b}$ ). The entrance of the knot-pusher in the thorax was well monitored by the gastroscope image, allowing the operator to recommend pressure adjustment or even knot removal when the knot was not correctly applied ( $\bullet$ Fig. 5c). The knots' edges were easily cut with the scissors ( $\bullet$ Fig. 5 d). As the anastomosis evolved, the benefit of the image provided by the gastroscope was gradually reduced, but the need for support and exposure was also less as the anastomosis was reaching completion. Although the aim was single-layer sutures involving both mucosa and muscle, we found that mucosa easily dislodged from the muscle layer and could be involuntarily missed by the needle; the gastroscope was very useful in monitoring the correct transmural involvement and in providing better exposure whenever necessary. The mean (standard deviation [SD]) time to perform complete anastomoses was 65.5 (10.9) minutes. The anastomoses were finally checked by their endoluminal aspect for lumen patency, intersuturing space and stitch mucosal misplacement. It was found in a few sutures that some stitches did not include the mucosal layer.

\section{In vivo studies}

The prone approach and the $\mathrm{CO}_{2}$ insufflation provided good exposure of the intrathoracic esophagus without the need for additional retraction instruments. This and the endoluminal transillumination of the esophagus allowed rapid access of the operative thoracoscope to select the esophageal segment of interest. In this study, we tested esophageal mobilization and esophagectomy using either a flexible gastroscope ( $\bullet$ Video 1 ) or rigid instruments ( Video 2 ), and both approaches were feasible. How-

\section{Video 1}

Gastroscopic esophagectomy. Steps of proximal section done with endoscopic submucosal dissection (ESD) knife. Esophageal dissection with a coagulation grasper. Once esophageal dissection was completed the distal esophagus was sectioned with a snare. The specimen was removed perorally. 

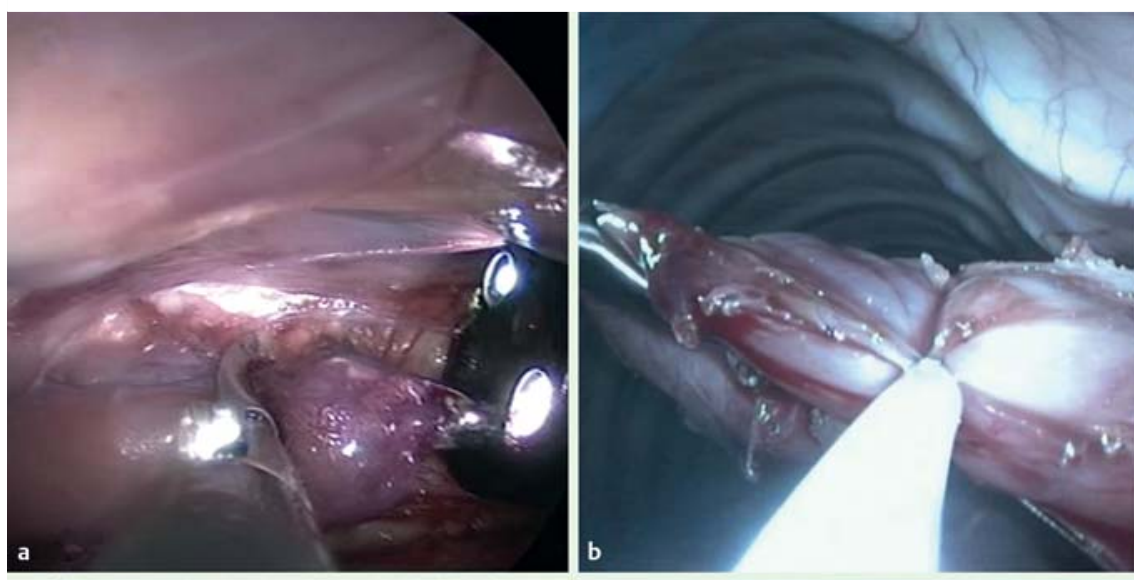

Fig. 6 In vivo segmental esophagectomy and esophago-esophageal anastomosis procedure: a esophageal segmentectomy (thoracoscope view); b esophageal segment being sectioned distally by the gastroscope snare (gastroscope view); c external final examination under endoscopic transillumination (thoracoscope view); $\mathbf{d}$ endoluminal examination of the anastomosis (gastroscope view).
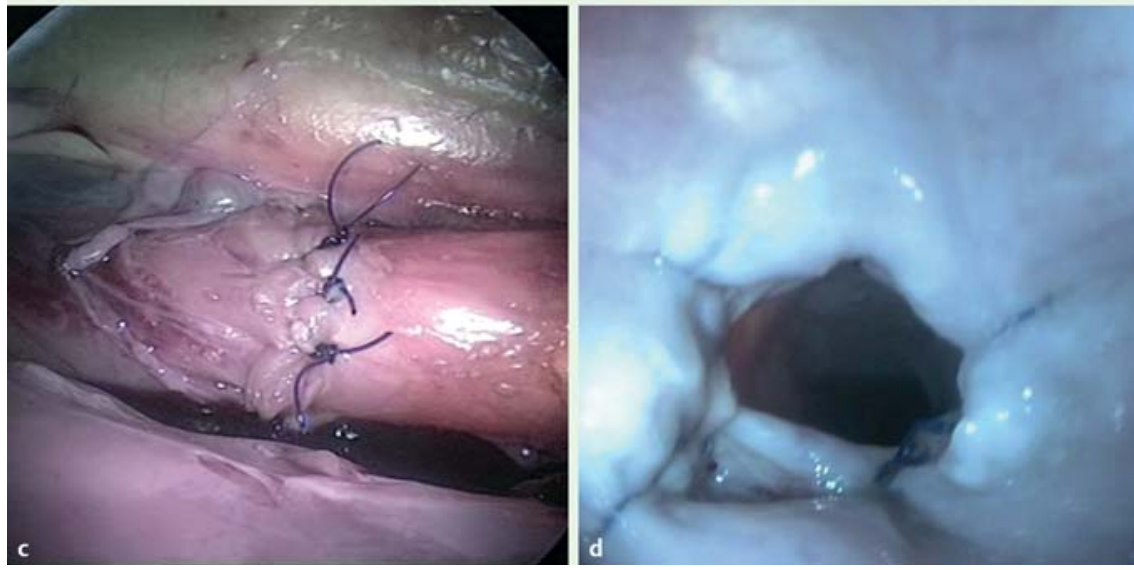

ever, the margin of the proximal esophageal stump was left more irregular when esophagectomy was performed using a flexible instrument (the gastroscope) than using a rigid instrument (the operative thoracoscope).

With both strategies, segmental esophagectomy was done and the specimen was removed perorally without complications ( Fig. $6 \mathrm{a}$ and $\mathbf{b}$ ).

In animals where esophagectomy was performed using rigid instruments (with a similar regularity of proximal and distal border), we performed esophageal anastomosis following the same principles as in the ex vivo training. Apart from some movement interference and some additional caution because of adjacent structures, the anastomoses were feasible and reproducible in all cases ( Video 3 ).

An interesting aspect of these experiments was that the thoracoscope view was sometimes not enough to see whether the needle position was the most appropriate for a specific suture orientation. However, combining the thoracoscope view with the gas-

\section{Video 2}

Operative thoracoscope esophagectomy. Using scissors through the operative thoracoscope working channel, and with slight movements of the gastroscope for esophageal traction, a safe blunt dissection of esophagus was carried out. The steps for esophagectomy are shown. The specimen was removed perorally.

\section{Video 3}

Esophageal anastomosis. Some steps of a complete single-layer, end-to-end esophago-esophageal anastomomosis in vivo, with images from a gastroscope and an operative thoracoscope sequentially intercalated. troscope view, it was always possible to correct the needle-holder position. The total mean operative time was 101.8 (32.9) minutes, including dissection, segmentectomy and anastomosis.

At the end of the procedure, the internal and external appearance of the anastomoses was checked ( $\bullet$ Video 4 ).

Externally, the thoracoscope was used to inspect the surface of the anastomosis by using endoluminal transillumination; the gastroscope was used to make small rotations of the anastomosis ( $\bullet$ Fig. 6c). Inside, all anastomoses were patent allowing distal passage of the endoscope, and almost all sutures incorporated the mucosa ( $\bullet$ Fig. $\mathbf{6 d}$ ). Air leak (bubbling) was detected in three animals. This most commonly occurred when the mucosa was not included in the stitch or when the distance between two stitches was too long. In these cases, we were able to correct it with additional stitching.

\section{Discussion}

$\nabla$

The esophagus is a fragile organ given its specific morphological characteristics and anatomical location, and it has been considered by surgeons something of a special zone [4]. This probably contributes to the slower dissemination of video-assisted surgery in the thorax when compared with laparoscopy [22,23]. Similar-

\section{Video 4}

Final checking of the esophageal anastomosis. The inside and outside appearance of the esophageal anastomosis.

online content including video sequences viewable at: www.thieme-connect.de/ejournals/abstract/endoscopy/ doi/10.1055/s-0030-1256012 
ly, transesophageal thoracic NOTES inspires less enthusiasm than abdominal NOTES.

Transesophageal NOTES endorses the possible absence of transthoracic incisions. Avoiding intercostal neuralgia, it brings the expectation of a potentially greater patient benefit in the thorax than in the abdomen [12]. Descriptions in a porcine model of transesophageal mediastinoscopy and thoracoscopy $[9,13]$, lung and pleura biopsy [13], lymphadenectomy [12,13], pericardial fenestration [12], vagotomy and esophagomyotomy [14], Heller myotomy [15], esophageal wall resection [16], with or without the help of endoscopic ultrasound (EUS), can be found in the literature. Besides the possible benefits, the procedure's main risks are always discussed: the 'blind' creation of the esophagotomy, the unpredictable thoracic side exit without EUS or fluoroscopic assistance, and finally the possible devastating consequences of leaking from an incomplete esophageal closure.

We hypothesized that a hybrid thoracic approach could achieve the benefits of reducing the number of transthoracic ports and simultaneously minimizing the limitations and risks of a pure transesophageal approach. This study explores the combination of the peroral route with a single transthoracic port for a complex intrathoracic procedure - segmental esophagectomy with esophagoesophageal anastomosis. The reduction of the usual four or five trocars used to a single transthoracic instrument was obtained as a result of three main factors: the use of an operative thoracoscope with a working channel for 5-mm instruments, the prone position instead of the regular left-lateral decubitus, and the coordinated beneficial use of the peroral gastroscope instruments.

The prone position is being promoted because it allows gravity to provide exposure with minimal handling, giving good esophageal visualization, simplifying dissection and reducing the operative times, without sacrificing patient safety [1]. In our in vivo experiments, we also confirmed these benefits with no need of any accessory port for lung retraction, and the single transthoracic instrument was totally focused on esophageal surgery.

The great improvement explored in this study is the help provided by the peroral/transesophageal port. The benefits start with the endoscopic transillumination of the esophagus by the thoracoscope; this step may be particularly helpful for direct visualization of a possible lesion area, orienting a more precise resection [24]. In this study, esophageal dissection and mobilization were carried out through two distinct strategies led either by a flexible gastroscope or by rigid thoracoscope instruments. Both strategies were successful in mobilizing, sectioning and dissecting the esophageal segment. When these processes were led by the gastroscope instruments, esophageal dissection and distal section were quite reliable, but the proximal esophageal section often left the stump margin too irregular. In contrast, esophageal dissection and section carried out by the thoracoscope instruments was reliable and rapid, and both esophageal margins were similar and quite regular. This was our main reason for testing the feasibility of the esophageal anastomosis in those animals where esophageal dissection and sectioning were carried out by the operative thoracoscope. It should be emphasized that even in this strategy, coordinated movements of the endoscope and traction were important for the operative thoracoscope-oriented work. In minimally invasive esophagectomy, specimen retrieval is currently performed via a separated neck incision, but in our experiments the specimen was always removed perorally; depending on its size, we predict this can be done in humans too.

The more impressive contribution of the gastroscope was during the creation of the anastomosis. The first stitch, which in a con- ventional thoracoscopic procedure is frequently the most difficult, was in our experience more straightforward because of the posterior esophageal wall grasping and proximal traction provided by the gastroscope instruments. Furthermore, the exposure of tissue layers, the countertraction and the capability for needle retrieval from and presentation to the operative thoracoscope whenever necessary were (apart from some inadequacy of the endoscopic forceps to grasp the needle securely) good technical potentialities revealed during experimentation. All in vivo anastomoses had at least 10 sutures, and each stitch was tied with five knots, with total control of the knotting by the gastroscope, from the thoracic entrance until its tissue adjustment. We found episodically that mucosa retracted and was missed during the stitch passage, but we could ameliorate this with increasing skills in coordination of tissue exposure and thoracoscope movements. Another advantage of a peroral access is that it is possibile immediately to check the luminal patency of the anastomosis and identify suspected areas of weakness needing additional stitches. In addition, endoscopic delivery of conditioning agents at this time can facilitate healing, as has been proposed previously [25].

As potential indications for this approach we would imagine benign lesions such as leiomyomas, resistant strictures, gastrointestinal stromal tumors, esophageal ruptures, complicated foreign bodies, esophageal atresia and even some selected nonadvanced malignant esophageal diseases. Initial series proposed minimally invasive methods, essentially for selected patients with earlystage esophageal cancer (stage II and below) [26]. Excluding high grade dysplasia and intramucosal cancer where organ preservation by endoscopic mucosal resection might be preferred, and excluding tumors of the esophagogastric junction that should have an abdominal transhiatal approach, we envision the possible application of hybrid thoracic NOTES in T1sm-T2 lesions even with thoracic lymphadenectomy. In this matter, recent reports support that sentinel node mapping, pre- or peri-intervention, can not only offer proper staging but also tailor an individualized surgical strategy and help to plan the field of irradiation $[4,27]$. Thus we predict the usefulness of our strategy for endoluminal lesion location, endoscopic submucosal injection of an agent with real-time thoracoscopic monitoring for sentinel node, followed by a posterior combined dissection lymphadenectomy with peroral removal.

Moreover, this approach could contribute to eliminating most of the current limitations of pure transesophageal NOTES that are potentially applicable to other thoracic extraesophageal conditions, namely in all those procedures previously attempted by a thoracic NOTES approach. The combination of the peroral approach with one transthoracic access seems advantageous because of the availability of two distinct images of the same procedure, resulting in a more accurate and safe assessment of the intervention, mainly an anastomosis, in such a delicate space as the thoracic cavity. We also believe that a reduction of the number of transthoracic ports to one is the maximum that we can expect in minimally invasive thoracic surgery for now. In fact, conventionally it is required to leave in place an external drain after any thoracic intervention in humans; this highlights the pertinence of our transthoracic access.

We would like to point out that our pig model has a healthy esophagus with no adhesions, and it is more elastic than human esophagus. Also, as a limitation, it might be emphasized that this was not a survival study. Thus, it is not possible to verify the safety of the approach, although we believe this approach has a good chance of being translated to humans in a stepwise reduction of 
transthoracic trocars, once there is secure endoscope sterility and the use of an oropharyngeal overtube.

In conclusion, this study confirms the feasibility of a hybrid peroral transesophageal approach with a single transthoracic trocar to carry out segmental esophagectomy with complete single-layer, end-to-end intrathoracic esophageal anastomosis in a porcine model. Moreover, this study reinforces the logic and pertinence of using a transesophageal approach to the thoracic cavity.

Competing interests: Jorge Correia Pinto is a consultant to Karl Storz.

\section{Institutions}

1 Surgical Sciences Research Domain, Life and Health Sciences Research Institute (ICVS), School of Health Sciences, University of Minho, Braga, Portugal

2 Department of Gastroenterology, Hospital de Braga, Braga, Portugal

3 Department of Internal Medicine, Centro Hospitalar do Médio Ave, Vila Nova de Famalicão, Portugal

4 Department of Oncology, Centro Hospitalar do Alto Ave, Guimarães, Portugal

${ }^{5}$ Department of Gastroenterology, Hospital de Sao Joao, Porto, Portugal

${ }^{6}$ Department of Pediatric Surgery, Hospital de Sao Joao, Porto, Portugal

\section{References}

1 Fabian T, Martin J, Katigbak $M$ et al. Thoracoscopic esophageal mobilization during minimally invasive esophagectomy: a head-to-head comparison of prone versus decubitus positions. Surg Endosc 2008; 22: $2485-2491$

2 Cuschieri A, Shimi S, Banting S. Endoscopic oesophagectomy through a right thoracoscopic approach. J R Coll Surg Edinb 1992; 37: 7-11

3 Shichinohe T, Hirano S, Kondo S. Video-assisted esophagectomy for esophageal cancer. Surg Today 2008; 38: 206-213

4 Perretta S, Allemann P, Dallemagne B et al. Natural orifice transluminal orifice surgery (NOTES) for neoplasia of the chest and mediastinum. Surg Oncol 2009; 18: 177-180

5 Kalloo AN, Singh VK, Jagannath SB et al. Flexible transgastric peritoneoscopy: a novel approach to diagnostic and therapeutic interventions in the peritoneal cavity. Gastrointest Endosc 2004; 60: 114- 117

6 Lima E, Rolanda C, Pego JM et al. Transvesical endoscopic peritoneoscopy: a novel $5 \mathrm{~mm}$ port for intra-abdominal scarless surgery. J Urol 2006; 176: $802-805$

7 Decker A. Culdoscopy a method for visual diagnosis of gynecologic disease. Clin Symp 1952; 4: 201 - 210

8 Fong DG, Pai RD, Thompson CC. Transcolonic endoscopic abdominal exploration: a NOTES survival study in a porcine model. Gastrointest Endosc 2007; 65: 312 - 318

9 Sumiyama K, Gostout CJ, Rajan E et al. Transesophageal mediastinoscopy by submucosal endoscopy with mucosal flap safety valve technique. Gastrointest Endosc 2007; 65: 679-683
10 Lima E, Henriques-Coelho T, Rolanda C et al. Transvesical thoracoscopy: A natural orifice translumenal endoscopic approach for thoracic surgery. Surg Endosc 2007; 21: 854-858

11 Willingham FF, Gee DW, Lauwers GY et al. Natural orifice transesophageal mediastinoscopy and thoracoscopy. Surg Endosc 2008; 22: 1042 1047

12 Fritscher-Ravens A, Patel K, Ghanbarri A et al. Natural orifice transluminal endoscopic surgery (NOTES) in the mediastinum: long-term survival animal experiments in transesophageal access, including minor surgical procedures. Endoscopy 2007; 39: 870-875

13 Gee DW, Willingham FF, Lauwers GY et al. Natural orifice transesophageal mediastinoscopy and thoracoscopy: a survival series in swine. Surg Endosc 2008; 22: 2117-2122

14 Woodward T, McCluskey D, Wallace MB et al. Pilot study of tranesophageal endoscopic surgery: NOTES esophagomyotomy, vagotomy, lymphadenectomy. J Laparoendosc Adv Surg Tech 2008; 18: 743 - 745

15 Pauli EM, Mathew A, Haluck RS et al. Technique for transesophageal endoscopic cardiomyotomy (Heller myotomy): video presentation at the Society of American Gastrointestinal and Endoscopic Surgeons (SAGES). Surg Endosc 2008; 22: 2279-2280

16 Fritscher-Ravens A, Cuming T, Jacobsen B et al. Feasibility and safety of endoscopic full-thickness esophageal wall resection and defect closure: a prospective long-term survival animal study. Gastrointest Endosc 2009; 69: $1314-1320$

17 ASGE, SAGES. ASGE/SAGES Working Group on Natural Orifice Translumenal Endoscopic Surgery White Paper. Gastrointest Endosc 2005; 63: $199-203$

18 Asakuma M, Perretta S, Alleman P et al. Challenges and lessons learned from NOTES cholecystectomy initial experience: a stepwise approach from the laboratory to clinical application. J Hepatobiliary Pancreat Surg 2009; 16: 249-254

19 Sodergren $\mathrm{MH}$, Clark J, Athanasiou $\mathrm{T}$ et al. Natural orifice transluminal endoscopic surgery: critical appraisal of applications in clinical practice. Surg Endosc 2009; 23: 680-687

20 Rolanda C, Lima E, Pego JM et al. Third-generation cholecystectomy by natural orifices: transgastric and transvesical combined approach. Gastrointest Endosc 2007; 65: $111-117$

21 Rolanda C, Lima E, Silva D et al. In vivo assessment of gastrotomy closure by over-the-scope-clips in an experimental model for varicocelectomy. Gastrointest Endosc 2009; 70: 1137-1145

22 Song SY, Na KJ, Oh SG et al. Learning curves of minimally invasive esophageal cancer surgery. Eur J Cardiothorac Surg 2009; 35: 689-693

23 Luketich JD, Alvelo-Rivera M, Buenaventura PO et al. Minimally invasive esophagectomy: outcomes in 222 patients. Ann Surg 2003; 238: 486 495

24 Malek MM, Shah SR, Katz AL et al. Endoscopically guided thoracoscopic esophagectomy for stricture in a child. Surg Endosc 2009; [Epub ahead of print]:

25 Reavis MK. The esophageal anastomosis: how improving blood supply affects leak rate. J Gastrointest Surg 2009; 13: 1558 - 1560

26 Law S. Minimally invasive techniques for esophageal cancer surgery. Best Pract Res Clin Gastroenterol 2006; 20: 925 - 940

27 Cense HA, Van Eijck CH, Tilanus HW. New insights in lymphatic spread of esophageal cancer and its implications for the extent of surgical resection. Best Pract Res Clin Gastroenterol 2006; 20: 893-906 